\title{
SUPERNOVA REMNANTS AND THE ISM: CONSTRAINTS FROM COSMIC-RAY ACCELERATION
}

\author{
Amri Wandel \\ Center of Space Science and Astrophysics \\ Stanford University, ERL \\ Stanford, CA 94305 USA
}

\begin{abstract}
.
Supernova remnants can reaccelerate cosmic rays and modify their distribution during the cosmic ray propagation in the galaxy. Cosmic ray observations (in particular the boron-to-carbon data) strongly limit the permitted amount of reacceleration, which is used to set an upper limit on the expansion of supernova remnants, and a lower limit on the effective density of the ISM swept up by supernova shocks. The constraint depends on the theory of cosmic ray propagation: the standard Leaky Box model requires a high effective density, $>1 \mathrm{~cm}^{-3}$, and is probably inconsistent with the present picture of the ISM. Modifying the Leaky Box model to include a moderate amount of weak-shock reacceleration, a self consistent solution is found, where the effective density in this solution is $\approx 0.1 \mathrm{~cm}^{-3}$, which implies efficient evaporation of the warm ISM component by young supernova remnants, during most of their supersonic expansion.
\end{abstract}

\section{INTRODUCTION}

If supernova remnants occupy a large fraction of the galaxy (McKee and Ostiker 1977, hereafter MO) additional acceleration by weaker shocks of supernova remnants, distributed over cosmic ray residence time in the galaxy is unavoidable. Detailed model calculations of cosmic ray acceleration by supernova shocks (Blandford and Ostriker 1980; Frannson and Epstein 1980) show that the resulting spectrum is consistent with the observed cosmic ray spectrum. Distributed acceleration cannot be a major source of cosmic ray energy, because it leads to a positive age-energy correlation, which is rejected by the observations. Even a relatively small amount of reacceleration can alter the cosmic ray spectrum significantly, in particular the energy dependence of the secondary-to-primary cosmic ray ratio (e.g. boron/carbon, hereafter B/C). It has been shown (e.g. Eichler 1980; Cowsik 1986) that observations of this ratio strongly constrain the acceptable amout of reacceleration . Wandel et. al. (1987, hereafter WELST) developed a model in which reacceleration of cosmic rays is treated self consistently, showing that a moderate amount of reacceleration by weak shocks (Mach number $<2$ ) is consistent with cosmic ray observations. We show that the constraint on reacceleration derived from $\mathrm{B} / \mathrm{C}$ ratio leads to constraints on the expansion of supernova remnants in the interstellar medium, and on the effective density of the ISM, felt by supernova remnants .

\section{ACCELERATION OF COSMIC RAYS BY SUPERNOVA REMNANTS}

Shock acceleration transforms a group of upstream particles with momentum $p_{0}$ into a (differential) power law distribution $J(p) \propto p^{-q}$ with an exponent (shock index)

$q=\left(2 M^{2}+2\right) /\left(M^{2}-1\right), \quad \gamma=5 / 3$ where $M$ is the Mach number of the shock.

Consider supernova remnants with a shock index $q$, corresponding to a Mach number $M_{q}=u_{q} / c_{s}$, where $u_{q}$ is the shock velocity and $c_{s}$ is the sound speed in the interstellar medium. In the initial adiabatic expansion phase, supernova remnants (and consequently the probability to encounter them) are small, and their contribution to reacceleration is negligible. In thehomologous expansion (Sedov-Taylor) phase the radius of the shock expanding in a uniform medium of density $\rho$, is given by (e.g. Spitzer 1968) $r=(2.1 E / \rho)^{1 / 5} t^{2 / 5}=12.7 n^{-1 / 5} t_{4}^{2 / 5} \mathrm{pc}$, where $\rho=1.3 m_{H} n, t=10^{4} t_{4} \mathrm{yr}$ is the remnant's age, and $E$ is the explosion energy; here and in the following we assume $E=10^{51} \mathrm{erg}$ 
(for different values our results scale with $E$ in the same manner as they do with $n^{-1}$ ). In terms of the shock velocity $u$ we have $r_{q}=36 n^{-1 / 3} u_{q, 2}^{-2 / 3} \mathrm{pc}$, and $t_{q}=1.3 \times 10^{5} n^{-1 / 3} u_{q, 2}^{-5 / 3} \mathrm{yr}$, where $u_{q}=10^{2} u_{q, 2} \mathrm{~km} \mathrm{~s}^{-1}$. Expansion in a cloudy medium is modified by cloud evaporation (MO). In the early, evaporation dominated phase, the expansion behaves as $r \propto t^{3 / 5}$, due to the change in the effective density. However, as discussed in III, when evaporation is efficient, we may use the homogeneous medium expression, with a higher effective density. Finally, when the internal pressure drops to that of the ambient medium, the remnant stops expanding, reaching a radius (Shull 1987) $r_{s n r} \approx 50 E_{51}^{11 / 35} n^{-13 / 35} \mathrm{pc}$.

In the standard Leaky Box model (e.g. Omes and Protheroe 1983) cosmic rays escape from the galaxy after having traversed a column of interstellar matter (path length), which at a few $\mathrm{GeV} / \mathrm{n}$ has the mean value of $\sim 9-10 \mathrm{~g} \mathrm{~cm}^{-2}$. The residence time of cosmic rays in the galaxy estimated from the relative abundance of the unstable nucleus ${ }^{10} \mathrm{Be}$, produced by spallation is $\tau_{c r} \sim 10^{7} \mathrm{yr}$ at a few GeV/n (Garcia Munoz et. al. 1981).

In the presence of a reacceleration process which transforms a given initial distribution into a power law distribution, $p^{-q}$, the steady state differential distribution of cosmic rays can be described by the equation (cf. WELST)

$$
J_{0}(p)-(R+S) J(p)=B\left[J(p)-(q-1) \int_{p_{0}}^{p} \frac{d p^{\prime}}{p^{\prime}}\left(\frac{p^{\prime}}{p}\right)^{q} J\left(p^{\prime}\right)\right]
$$

where $R, B$, and $S$ are the rates ${ }^{1}$ of escape, reacceleration and spallation, respectively, $J_{0}$ is the cosmic ray source spectrum, and $p_{0}$ is a cutoff due to ionization losses at low energies. The secondary distribution satisfies a similar equation, with the source term replaced by the primary distribution.

The B/C data from the HEAO-3 experiment (Engelmann et. al. 1983), strongly limit the allowed amount of reacceleration. In the frame of the standard leaky box (SLB) model $\left(R_{0}=0.11 \mathrm{~g}^{-1} \mathrm{~cm}^{2}\right)$, this limit is very stringent (eq. [2.b]). If reacceleration is taken into account self consistently, WELST find a less stringent constraint. Their best fit of the $\mathrm{B} / \mathrm{C}$ data compared to the constraint from the SLB with the same shock index gives (see Fig. 1)

$$
\begin{gathered}
B \approx(0.2 \pm 0.05) \mathrm{g}^{-1} \mathrm{~cm}^{2} \quad R_{0}=0.2 ; q=4(W E L S T), \\
B<0.03 \mathrm{~g}^{-1} \mathrm{~cm}^{2} \quad R_{0}=0.11 ; q=4(S L B) .
\end{gathered}
$$

At the energies under consideration the Larmor radius and the mean free path to pitch angle scattering, $\lambda$, of cosmic rays are small compared to the scale height of the galactic disk, $h_{d}$, and the diffusion approximation may be used. Over a time $t \gg \lambda / c$, an average cosmic ray covers a volume of radius $r_{c r}(t) \approx(D t)^{1 / 2}$, where $D=\lambda c / 3$ is the diffusion coefficient. During their lifetime in the galaxy cosmic rays at a few $\mathrm{GeV} / \mathrm{n}$ travel through a column of $R_{0}^{-1}$, which, assuming a mean density $n_{d}=1 \mathrm{~cm}^{-3}$, implies a residence time in the galactic disk of $\tau_{d}=\left(1.3 m_{H} n_{d} R_{0} c\right)^{-1} \approx 5 \times 10^{5} R_{0}^{-1} \mathrm{yr}$. An average cosmic ray propagates a linear distance of $d_{c r}=r_{c r}\left(\tau_{c r}\right)$ covering a portion of the disk of volume $V_{c r}=2 \pi h_{d} d_{c r}^{2}$. Assuming supernovae are distributed uniformly in the galactic disk ${ }^{2}$ the number of supernovae within this volume is $N_{s n}=\pi d_{c r}^{2} \tau_{d} \sigma$, where $\sigma=10^{-10} \sigma_{-10}$ supernovae $\mathrm{pc}^{-2} \mathrm{yr}^{-1}$ is the surface rate of supernovae in the galaxy $\left(\sigma_{-10}=0.6\right.$ corresponds to a total rate of one supernova every 20 years within a disk radius of $15 \mathrm{kpc}$ ).

1 These rates are expressed in inverse path length $\left(\mathrm{gram}^{-1}\right)$; in particular $B$ is the inverse pathlength between shocks, so that $B / R$ is the average number of shocks encountered during a cosmic ray residence in the galaxy.

${ }_{2}$ Type II supernovae are clustered in space and in time, but the clustering effect may increase the reacceleration rate over the rate produced by a uniform distribution, since presumably cosmic rays are initially accelerated by supernova shocks, and the clustering effect increases the probability to encounter a shock after leaving the parent supernova. 
A cosmic ray particle will encounter a supernova remnant of radius $r_{q}$ within a time $t_{q}$ if it happens to be within a sphere of radius $r_{q}+r_{c r}\left(t_{q}\right)$ about the supernova. Since cosmic rays are assumed to be homogeneously distributed in the volume $V_{c r}$, the probability for such an event is given by the ratio between the volume defined by this sphere and $V_{c r}, P_{c r}(q)=2\left[r_{q}+r_{c r}\left(t_{q}\right)\right]^{3} / 3 d_{c r}^{2} h_{d}$. The average number of shocks encountered by a cosmic ray particle during its residence time in the disk is given by $N_{s n} P_{c r}$. From the definition of the reacceleration and escape rates, this number is also given by the ratio $B / R$. Comparing the two expressions we have

$$
\begin{gathered}
B(q)=N_{s n} P_{c r}(q) R_{0}=1.5 \sigma_{-10} h_{d, 2}^{-1} r_{q, 2}^{3}\left[1+\frac{d_{c r}}{r_{q}}\left(\frac{t_{q}}{\tau_{c r}}\right)^{1 / 2}\right]^{3} \\
=0.07 \sigma_{-10} h_{d, 2}^{-1} n^{-1} C_{q}^{3} u_{q, 2}^{-2}
\end{gathered}
$$

where $C_{q}=1+0.3 d_{c r, 2} \tau_{c r, 7}^{-1 / 2}\left(n r_{q, 2}\right)^{1 / 4}=1+0.3 d_{c r, 2} \tau_{c r, 7}^{-1 / 2} n^{1 / 6} u_{q, 2}^{-1 / 6}, B$ is in units of $\mathrm{g}^{-1} \mathrm{~cm}^{2}$, and subindices denote obvious units. (As $P_{c r}$ is strongly peaked towards higher values of $q$, we have assumed the reacceleration due to supernova remnants with $q^{\prime} \leq q$ is dominated by the largest ones, having $q^{\prime} \approx q$ ).

\section{IMPLICATIONS}

Eqs. (2) and (3) yield a direct constraint on the expansion of supernova remnants. One can, however, use this result to constrain the effective density felt by the expanding remnant. The magnetosonic sound speed in the hot component ${ }^{3}$ (density $\left.n_{0}\right)$ of the ISM $\left(P / k=3 \times 10^{3}\right.$ and a magnetic field of $3 \mu G)$ is $c_{s}=\left(\gamma P_{g a s} / \rho+v_{A}^{2}\right)^{1 / 2} \approx 150\left(n_{0} / 3 \times 10^{-3} \mathrm{~cm}^{-3}\right)^{-1 / 2} \mathrm{~km} \mathrm{~s}^{-1}$. A shock index of $q=4(M=1.7)$ then corresponds to $u=250 \mathrm{~km} \mathrm{~s}^{-1}$.

If the effective density were close to the density of the hot component, the reacceleration rate is much too large; e.g. for $n=3 \times 10^{-3} \mathrm{~cm}^{-3}$ eq. (3) yields $B>2 \mathrm{~g}^{-1} \mathrm{~cm}^{2}$, which, as seen from Fig. 1 , grossly violates the $\mathrm{B} / \mathrm{C}$ data. However, cloud evaporation in the early expansion phases gives a higher effective density. Solving for $n$ eq. (3) gives

$$
\frac{n}{\left(1+0.8 n^{1 / 6}\right)^{3}} \approx 0.015 B^{-1} c_{s, 2}^{-2}
$$

where $c_{s, 2}$ is in units of $100 \mathrm{~km} \mathrm{~s}^{-1}$ and we have taken $\sigma_{-10}=0.6, h_{d, 2}=\tau_{c r, 7}=1$ and $d_{c r}=300 \mathrm{pc}$ (Note that eqs. (3) and (4) are only weakly dependent on the parameters of the cosmic ray model, $\tau_{c r}$ and $\left.d_{c r}\right)$. Combining this with the constraints on the reacceleration rate derived from the $\mathrm{B} / \mathrm{C}$ data, eqs. (6.a,b), we get $n \approx 0.1 \mathrm{~cm}^{-3}$ (WELST) and $n>0.8 \mathrm{~cm}^{-3}$ (SLB). The respective limits on the radius are $r(q=4) \approx 40 \mathrm{pc}$ and $r<20 \mathrm{pc}$.

Assuming once more a uniform supernova distribution, the porosity of hot supernova cavities in the ISM is

$$
Q=\left(\frac{\sigma}{2 h_{s n}}\right)\left(\frac{4 \pi}{3} r_{s n r}^{3}\right)\left(\frac{r_{s n r}}{v_{r m s}}\right) \approx 0.2 E_{51}^{1.26} n^{-1.5}
$$

where $h_{s n}$ (taken here as $300 \mathrm{pc}$ ) is the scale height of the supernova distribution and $v_{r m s} \approx 10 \mathrm{~km} \mathrm{~s}^{-1}$ is the random velocity of the interstellar gas. The filling factor of hot coronal gas is $f \approx Q /(1+Q)$. For an effective density $n \approx 0.1$ (WELST), $f$ is close to unity and our choice of the sound speed is justified. However, in the SLB scenario $f<0.25$, and the shock is effectively expanding in a much denser medium $\left(n_{0} \approx 0.1 \mathrm{~cm}^{-3}\right)$, corresponding to a lower sound speed, which gives a yet higher limit on $n$ (Wandel 1987). However, since the effective density cannot be larger than the average density in the disk, $1 \mathrm{~cm}^{-3}$, this case may probably be ruled out, making the SLB inconsistent

\footnotetext{
3 The density of the hot phase determines the sound speed as long as its filling factor is of order unity; the effective density, $n$, may however be much higher.
} 


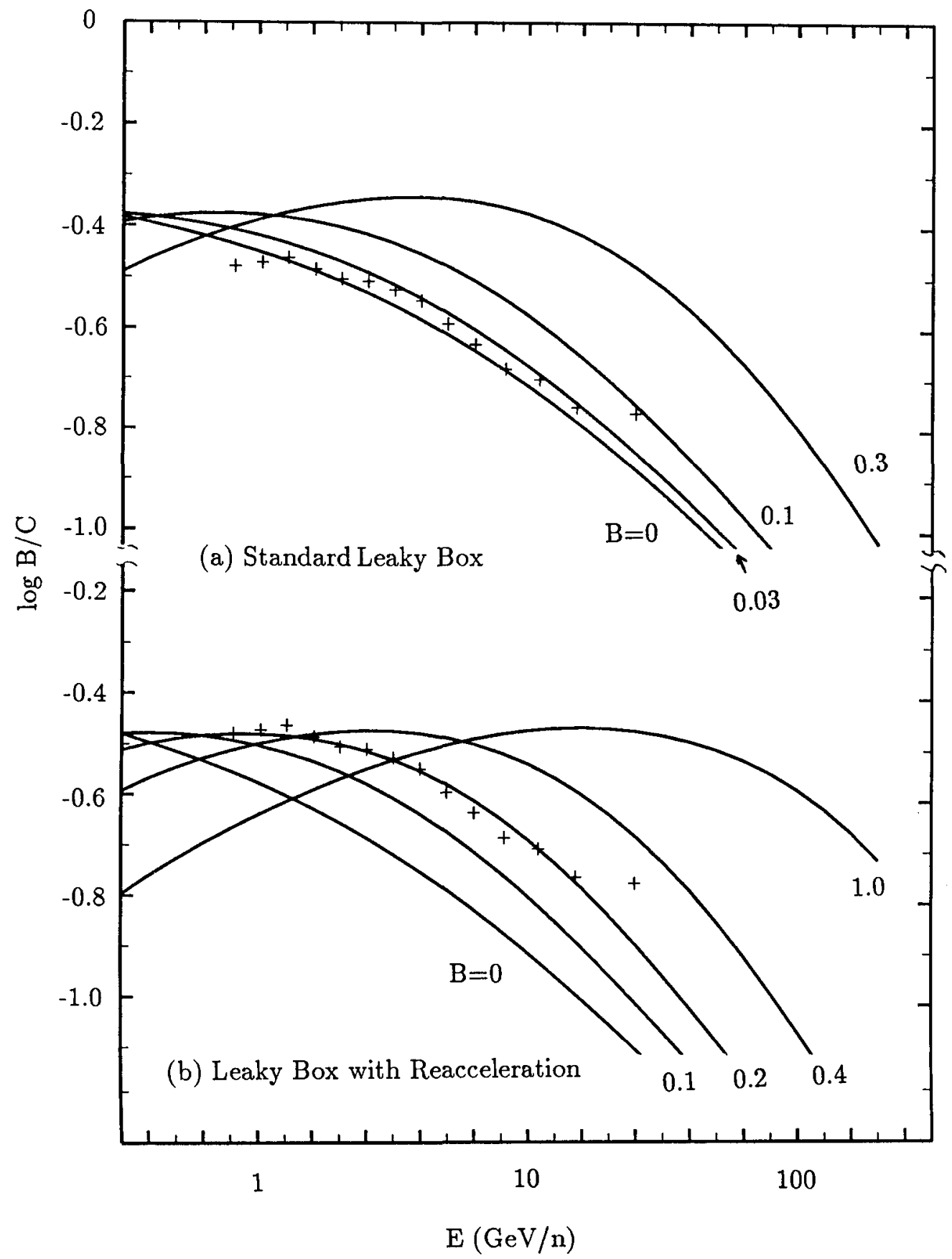

Fig 1 (a) The secondary-to-primary ratio (normalized to the Boron/carbon ratio) calculated in the standard Leaky Box model $(\mathrm{a}),\left(R_{0}=0.11, \alpha=0.6\right.$ and various amounts of reacceleration $(B=0$, $0.03,0.1,0.3$ ), and in the modified Leaky Box model (WELST) (b), with an increased escape rate, $R_{0}=0.2$ and shock acceleration index $q=4$. Curves correspond to no reacceleration $(B=0)$, $B=0.1,0.2,0.4$ and 1. Data points are from Engelmann (1983). 
with reacceleration by supernova remnants. In the MO model the early adiabatic expansion of supernova remnants is dominated by evaporation of clouds inside the remnant, rather than by sweeping up of the hot intercloud medium by the shock front. The expansion in this phase is given by $r \approx 12\left(E_{51} / n_{h}\right)^{-1 / 5} t_{4}^{2 / 5} \mathrm{pc}$, where $n_{h}$ is the gas density inside the remnant. As long as the evaporation of clouds is efficient, $n_{h}$ is much higher than the density of the hot intercloud medium, $n_{0}$. At later times the evaporation time becomes larger than the expansion time and the density in the gas inside the remnant approaches $n_{0}$. The transition is given by $n_{h} / n_{0}=1+\left(r / r_{e s}\right)^{-5 / 3}$, and occurs at the characteristic radius $r_{e s} \approx 10\left(E_{51} / a_{p c}\right)^{2 / 5} f_{c l}^{1 / 5} n_{0}^{-3 / 5} \mathrm{pc}$, where $a_{p c}$ and $f_{c l}$ are the clouds' radius (in parsec) and filling factor, respectively. As long as the evaporation is fast enough to ensure $n_{h} \approx n_{a v}$, we have the familiar homologous expansion with an effective density $n=n_{a v}$ (which is the approximation used in this work). This is the case for

$$
r<r_{e s}\left(n / n_{0}\right)^{-3 / 5} \approx 10\left(E_{51} / a_{p c}\right)^{2 / 5} f_{c l}^{1 / 5} n^{-3 / 5} \mathrm{pc}
$$

When $n_{h}$ drops, the expansion proceeds at a faster rate, $r \propto t^{\eta}, \quad \eta=3 / 5$. Numerical calculations (Cowie, McKee and Ostriker 1981) show that the expansion is actually intermediate between these two extremes.

For $n=0.1 \mathrm{~cm}^{-3}$ and warm clouds, eq. (6) gives $r<30 \mathrm{pc}$, which is roughly consistent with the constraint $(r(q=4) \approx 40 \mathrm{pc})$ derived from the reacceleration argument in the WELST model.

The SLB model, however, requires $n>1 \mathrm{~cm}^{-3}$, which must invoke evaporation of the cold clouds too, for which eq. (11) yields $r<6$ pc. Since this is much less than the reacceleration constraint $(\sim 20 \mathrm{pc})$, evaporation cannot keep the required high effective density long enough, and supernova remnants will expand too fast, producing too much reacceleration.

\section{REFERENCES}

Axford, W.I. 1981, 17th Int. Conf. Cosmic Rays (Paris).

Blandford,R.D. and Ostriker, J.P. 1980, Ap. J., 237, 793.

Cowie, L.L, McKee, C.F., and Ostriker, J.P. 1981, Ap. J., 247, 908.

Cowsik, R. 1986, Astr. Ap., 155, 344.

Eichler, D.S. 1980, Ap. J., 237, 809.

Engelmann , J.J., et. al. 1983, Proc. 18th Internat. Cosmic Ray Conf. (Bangalore), $2,17$.

Fransson, C. and Epstein, R.I. 1980, Ap. J., 242, 411.

Garcia-Munoz, M., Simpson, J.A. and Wefel, J.P. 1981, Proc. 17th Internat. Cosmic Ray Conf. (Paris) 2, 72.

McKee, C.F., and Ostriker, J.P. 1977 (MO), Ap. J., 218,, 148 .

Ormes, J. and Protheroe, R.J. 1983, Proc. 18th ICRC (Bangalore), 2, 221.

Shull, J.M. 1987, in Interstellar Processes, ed. D. Hollenbach and H. Thornson, Reidel.

Spitzer, L. 1968, in Diffuse Matter in Space p.199, John Wiley, New York.

Wandel, A., Eichler, D.S., Letaw, J.R., Silberberg, R., and Tsao, C.H. 1987 (WELST), Ap. J., 316, 676.

Wandel, A. 1987, in preparation. 